\title{
A survey on men's awareness of premenstrual syndrome and marital satisfaction in married women in Tehran, Iran
}

\begin{abstract}
Background:

The Premenstrual Syndrome (PMS) is a major clinical disorder with a number of temporary behavioral changes that is greatly affecting female population. Available information about PMS and marital satisfaction remains unclear, especially among women suffering from symptoms of PMS. The link between spousal supports has also been found to be related to marital satisfaction while no literature exists scrutinizing the effect of men's awareness of PMS on marital satisfaction. This study intended to examine men's awareness of PMS as a predictive factor for marital satisfaction among married women affected by PMS.
\end{abstract}

Aim:

To scrutinize the effect of men's awareness of Premenstrual Syndrome (PMS) on marital satisfaction among married PMS-affected women.

Methods:

The sample for this cross-sectional survey comprised of 277 married 19-49 years old women who registered at gynecological outpatient clinics in Tehran, Iran. Data were collected following a multistage random cluster sampling approach in 2015. Main research variables were demographic data, an adapted Premenstrual Syndrome Scale (PMSS), Enrich Marital Satisfaction Scale as well as men's awareness of the Premenstrual Syndrome.

Results:

This study found that the men's awareness of PMS and having PMS symptoms were correlated to, and at times anticipated, the likelihood of marital satisfaction among PMS-affected women.

Conclusions:

The results can assist frontline health workers and health promotion strategists seeking to improve women's health by providing visions into personal, relationship, and socio-cultural factors and offering consultation services for PMS issues in primary health care facilities in Iran.

Keyword: Female population; Marital satisfaction; Men's awareness; Premenstrual syndrome; Women's health; Survey 